\title{
Reflexiones a raíz de Filosofía para médicos, un texto de Mario Bunge
}

\author{
Reflections on Philosophy for physicians, \\ a text by Mario Bunge
}

Autor: Mario Bunge

Barcelona: Gedisa; 2012. 208 páginas

ISBN 978-84-9784-739-1

Silva Ayçaguer, Luis Carlos ${ }^{1}$

El propósito que me anima es reflexionar en torno a los contenidos del libro Filosofía para médicos (1) del afamado epistemólogo argentino Mario Bunge, publicado recientemente en España y motivo de diversas conferencias y entrevistas realizadas en diciembre de 2012. No se trata por tanto de una recensión clásica sino de opinar sobre las ideas que el libro abarca o sugiere. Por el amplio diapasón de temas que aborda y la naturaleza polémica de muchos de ellos, se trata de un material de mucho interés para el personal sanitario.

Desde mi punto de vista, el material irradia luz pero proyecta algunas sombras que merecen análisis crítico. Ese análisis responde a la invitación a polemizar, implícita en su advertencia de que "en las ciencias la duda es preferible a la firme creencia en dogmas" (1 p.91). Mi propósito no es hacer un repaso exhaustivo del libro. Si la presente nota resulta algo extensa no es porque sean muchos los puntos con los que discrepo, sino porque la fundamentación de la mayoría de tales disensos convoca a la reflexión pausada y responsable, al examen de antecedentes y a no desoír la advertencia que nos hace el propio Bunge cuando señala que determinadas críticas destructivas pueden ser irresponsables (1 p.169).

Filosofía para médicos es un libro que esclarece asuntos trascendentes, derriba mitos y recuerda no pocas verdades que todos los profesionales de la salud, los médicos en particular, harían bien en co-

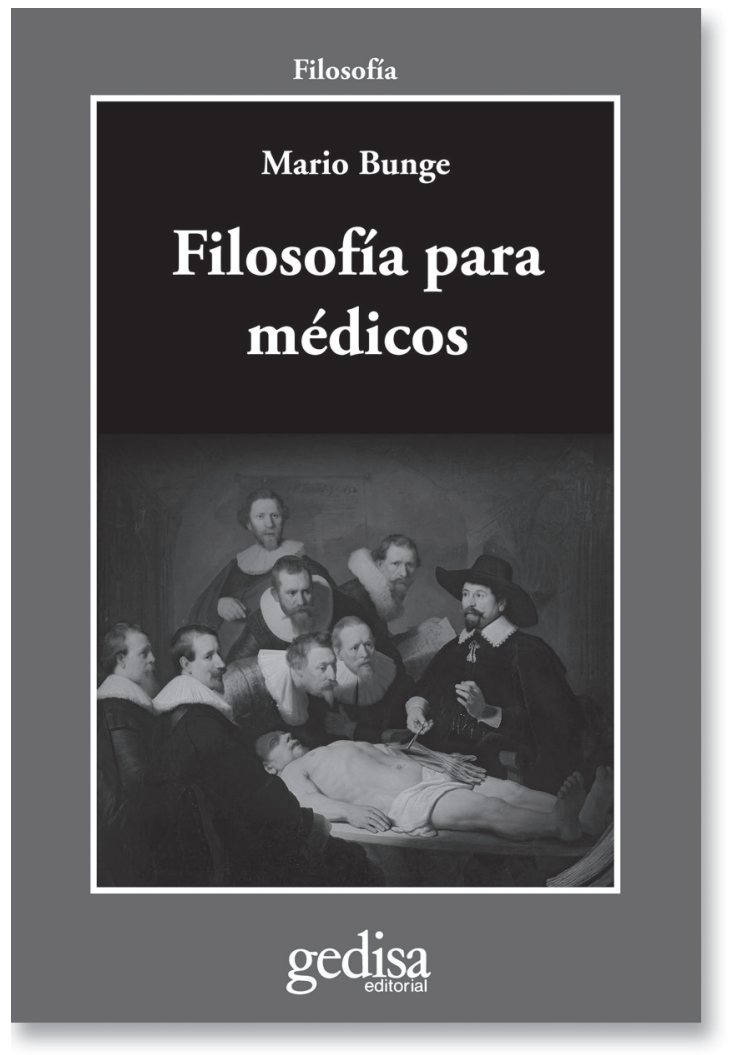

nocer. Lamentablemente, sin embargo, contiene mensajes que resultan confusos, se resiente de algunos prejuicios e incurre en varios errores. El presente texto destaca los méritos, pero no elude el examen de sus problemas fundamentales, con lo cual espero contribuir a un tema de notable trascendencia. 


\section{LOS ACIERTOS DE BUNGE}

Entre los méritos más notables de la obra se halla la desenvoltura expositiva y la sencillez con que el autor transmite ideas relativamente complejas. Bunge despliega un lenguaje elegante, sin rebuscamientos, que ameniza la lectura; y, a la vez, vuelca en él su enorme erudición, tanto a través de ejemplos precisos y oportunos, como mediante un considerable caudal de informaciones históricas y precisiones filosóficas y metodológicas de valor inestimable.

La crítica, sin fisuras ni medias tintas, a la que se someten las pseudociencias, tiene especial merecimiento. Si bien es algo que Mario Bunge ha tratado en muchas de sus obras precedentes, sus observaciones sobre las falsas panaceas y la denuncia de terapias fraudulentas, tales como la homeopatía y el curanderismo, se erigen como sólido y fundamentado desenmascaramiento de prácticas médicas ajenas a la ciencia. Pero también revelan los riesgos a que está sometida la praxis sanitaria cuando quienes la ejercen desdeñan la importancia de someter sus convicciones al juicio que puedan merecer tales procedimientos desde una perspectiva filosófica y dentro de un marco metodológico adecuado.

En última instancia, subraya Bunge una y otra vez, nada supera al dictamen derivado de la experiencia objetiva y especialmente de los ensayos clínicos, en aquellas áreas que los consientan. Particularmente sugestiva resulta su propuesta de conceptuarlos no solo como una "prueba de oro" -algo con lo que poca gente discrepa- sino de considerarlos como una condición necesaria para alcanzar un escalón superior en la gama de recursos para la generación de nuevos conocimientos médicos (1 p.40). Aludo a la que él Ilama "prueba de platino", que se provee cuando, además de verse respaldados por dicha prueba de oro, los argumentos se asientan en explicaciones de los mecanismos que le permitieron superarla.

La comprensión del papel del efecto placebo, así como la importancia medular de la plausibilidad teórica de las hipótesis, las falacias que se derivan de la transgresión del pensamiento lógico y la necesidad de hallar, en la medida de lo posible, los mecanismos causales de los procesos asociados a la salud y la en- fermedad atendiendo a la naturaleza sistémica que les caracteriza, figuran como un leitmotiv en todo el material.

Especial destaque reclama el encuadre social en que ha de ubicarse la práctica sanitaria. Aunque la comprensión de la naturaleza hondamente social del desempeño del médico es ya de muy larga data y ha sido muy estudiada por prestigiosas figuras, especialmente desde la década de 1950, Bunge lo resalta de manera acertada y oportuna. Con notable capacidad de síntesis, el autor establece la diferencia entre lo legal y lo moral, así como el conflicto que puede darse entre el legítimo afán de bienestar personal de un profesional de la salud, o los réditos de una empresa que opere en este ámbito, y el compromiso que uno y otra han de asumir con la verdad y con las personas a quienes supuestamente han de beneficiar.

\section{ALGUNOS ERRORES PUNTUALES}

Algunas afirmaciones del libro son simplemente erróneas. Quizás no tengan mucha importancia intrínseca, pero a la luz del tono tan tajante de las afirmaciones que pueblan el libro, resultan, cuando menos, enojosas. Veamos algunos ejemplos.

Se dice que la hipótesis diagnóstica implícita en el silogismo "si el paciente exhibe el signo o indicador objetivo S, entonces es posible que padezca el trastorno $E^{\prime \prime}$ se funda en investigaciones biomédicas, "en particular en ensayos clínicos" (1 p.16). Esto es erróneo, porque los experimentos en humanos no se usan para identificar indicadores objetivos propios de una enfermedad sino para valorar efectos de posibles intervenciones médico-sanitarias, como el propio Bunge puntualiza cuando aclara: "El objetivo central de los ensayos clínicos es identificar los efectos reales causados por el tratamiento que se ensaya" (1 p.131). Los elementos empleados para el diagnóstico pueden ser causas de la enfermedad (como la presencia de un virus a los efectos de desarrollar dengue) o manifestaciones derivadas de ellas (como fiebre o prurito cutáneo en esa misma dolencia). Los elementos causales quedan excluidos por razones éticas, ya que no es aceptable que se induzcan 
en un grupo experimental para registrar si la aparición de la enfermedad es más acusada en dicho grupo que en aquel que no fue objeto de tal intervención. Las manifestaciones que subsiguen a la instalación de la dolencia tampoco pueden ser evaluadas por esa vía debido a que los ensayos clínicos son necesariamente prospectivos: no se pueden crear grupos con y sin dichas manifestaciones para examinar la aparición del trastorno en ellos, que es anterior.

En la propia esfera del diagnóstico se lee que, si se observa una tos refractaria, se podrá sospechar una tuberculosis y que una radiografía o un análisis de sangre permitirán confirmar o refutar de manera prácticamente concluyente el mecanismo. Y agrega: "Se habrá dado con una causa, la que es condición a la vez necesaria y suficiente para que ocurra el efecto o signo en cuestión" (1 p.88). ¿De qué mecanismo habla? ¿Cuál es la condición necesaria y suficiente para que ocurra la tos refractaria? Los medios diagnósticos mencionados podrían haber revelado la presencia del bacilo de Koch, por ejemplo, pero esta no es necesaria para que se produzca la tos (muchos otros agentes pueden producirla), ni es suficiente, pues no todos los portadores del bacilo tienen tos.

En otros puntos del libro, Bunge pone más ejemplos equivocados en relación con las categorías de necesidad y suficiencia. Menciona que la desnutrición infantil es condición necesaria y suficiente para el retardo en el desarrollo (1 p.143); pero la realidad es que tal condición ni es necesaria (la desnutrición puede aparecer como consecuencia de una ingesta deficiente en un niño cuyo desarrollo haya sido perfectamente normal), ni es suficiente (un niño puede tener un retardo en el desarrollo psicomotriz e incluso físico debido a problemas neurológicos que nada tienen que ver con la nutrición). Algo parecido ocurre con la deshidratación y la muerte. Aquella no es causa necesaria y suficiente para la muerte, como se afirma. Es obvio que se puede fallecer sin que necesariamente se haya producido deshidratación, como ocurre en los accidentes de tránsito o con una sobredosis de opiáceos.

Finalmente, un detalle, acaso debido a una inadvertencia: se califica el hecho de que "siempre hay dos desenlaces posibles para toda enfermedad: recuperación o muerte" (1 p.163) como uno de los dos grandes descubrimientos conocidos por el médico hipocrático. Puesto que este aserto no se pone en duda, Bunge está de hecho convalidándolo. Desde luego, no es así. Mucha gente no se recupera nunca de la enfermedad (es el caso, por ejemplo, de la retinosis pigmentaria, la psoriasis o la artritis) sin que ello lo lleve a la muerte.

\section{DESCALIFICACIONES SUPERFICIALES}

Bunge suele ser extremadamente tajante, sumario, definitivo. Raramente -quizás nunca- usa giros tales como "a mi juicio", "desde mi punto de vista", "estimo que". Es su estilo. A veces se echa en falta la alusión a opiniones discrepantes con sus puntos de vista que pudieran o debieran ser tenidas en cuenta. Pero lo justo, por lo general, es que al examinar un texto, nos remitamos solo a lo que se dice, sin recriminar al autor lo que omite (a).

Ahora bien, dentro de ese estilo, el texto enarbola descalificaciones globales que, de un plumazo, trivializan la obra de importantísimos e influyentes pensadores en el campo de la filosofía o el pensamiento médico. En este sentido, la obra se alinea por momentos con la tendencia actual a la frivolización de la cultura (2) (en este caso, de su vertiente científica y filosófica), haciendo concesiones facilistas que resultan chocantes en una obra con el vuelo y el rigor que exige el tema tratado.

Por ejemplo, a juicio de Bunge, "el médico filosofa todo el día" (1 p.13) mientras que ni Friedrich Nietzsche ni Martin Heidegger Ilegaron a ser filósofos, sino meros "escritores parafilosóficos" quienes "al despotricar contra la racionalidad y la moralidad" solo hicieron un aporte: su contribución al nazismo (1 p.123). Lo menos que puede decirse es que esta lapidaria sentencia no es seria, que transmite una imagen caricatural y parcializada sobre ambos intelectuales. Si bien ellos despiertan reticencias en el plano político debido a que las ideas del primero (quien murió 30 años antes de que viera la luz el nazismo) fueran usadas por el régimen nacional-socialista, y a que el segundo fue esquivo en la crítica a dicho régimen, el cual también "usó" parte de sus teorías, se trata de pensadores de enorme estatura, cuya obra estrictamente filosófica es colosal, por lo cual es objeto de estudio por los más destacados especialistas en el mundo entero. 
No me extenderé en datos que en cierto sentido son laterales, pero baste decir que algunas de las ideas de Nietzsche (también su poesía y su obra filológica) se hallan entre las más notables y sugerentes del siglo XIX, mientras que la obra de Heidegger es simplemente monumental, y dejó una marca en las avenidas del quehacer filosófico posterior, no solo para la intelectualidad germana y anglosajona, sino para filósofos imprescindibles de la Europa latina, como Jean-Paul Sartre, Miguel de Unamuno y José Ortega y Gasset.

No soy un experto en psicología ni mucho menos en psicoanálisis. Sospecho fuertemente, no obstante, que a Bunge le asiste por entero la razón cuando señala algunas zonas a todas luces delirantes de la teoría psicoanalítica originalmente expuesta por Sigmund Freud y luego desarrollada $-\mathrm{y}$ explotada- por sus seguidores. No en balde Karl Popper observó que las teorías de Freud eran no falsables, lo cual le permitía reinterpretar los datos para mantener las hipótesis aunque carecieran de respaldo empírico.

Pero de ahí a descalificar en bloque toda la obra de Freud, va un trecho considerable. Bunge puede tener la convicción de que este pensador e investigador fue "el peor psicólogo del siglo" (1 p.91), pero este tipo de expresiones dejan la impresión de que le anima más la emoción que el examen pausado de la realidad y de la historia. A juicio de Bunge, quienes resultaron engatusados por él lo fueron por ignorancia o por afán de lucro. Tengo la impresión de que las cosas no son tan simples. No creo, por ejemplo, que la Clark University (Worcester, Massachusetts) haya conferido a Freud el doctorado honoris causa (1930) por esas razones. Pero todo eso es cuestión de opinión y la de Bunge es muy respetable. Afirmar, sin embargo, que las telarañas freudianas fueron barridas por "el vendaval de psicofármacos, los cuales reemplazaron a los mitos psicoanalíticos" entraña una falacia.

Si bien la afirmación acerca de que los mitos de Freud han sido barridos es en gran medida correcta, a pesar de que sus doctrinas aún se estudian en muchas facultades y escuelas de psicología (aunque con frecuencia solo para criticarlas), y de que en medida no despreciable sobrevivan, al menos en los reductos "lacanianos", lo fallido reside en que no cabe atribuir ese mérito a los fármacos sino a una reflexión sistematizada desde una perspectiva científica. Muy especialmente, a las contribuciones de brillantes pensadores y estudiosos a lo largo del siglo pasado, e incluso más recientemente, como es el caso de Michel Onfray (3).

Ningún vendaval psicofarmacológico ha barrido, por ejemplo, a la orientación cognitivoconductual de la psicoterapia, hoy dominante. El problema es que estamos ante una manifestación de deslumbramiento implícito por los psicofármacos, algunas de cuyas expresiones han sido duramente cuestionadas a la vez que millones de personas han resultado embaucadas por las argucias derivadas de la codicia de sus promotores (4). Esta fascinación acrítica se incrementa cuando las víctimas son irresponsablemente desinformadas por los médicos (5), o cuando los propios prescriptores son manipulados hasta el punto de que Ilegan a "creer que las drogas son mucho más efectivas de lo que realmente son para dar lugar a un sobretratamiento masivo de antipsicóticos y antidepresivos", como documentaba recientemente Peter Gøtzsche en un artículo aparecido en la prestigiosa revista Trials (6).

A los psicofármacos le debemos mucho, pero me temo que el "vendaval" psicofarmacológico, en particular el de los más famosos, los inhibidores selectivos de la recaptación de la serotonina (tales como la fluoxetina y la paroxetina) (7), ha hecho muchísimo más daño que Freud. Según se ha documentado con todo rigor (8), no solo no existe corroboración alguna de que los niveles bajos de serotonina produzcan desorden mental alguno, sino que se cuenta con evidencias significativas en contra de dicha hipótesis, aparte de otros serios daños colaterales producidos por estos fármacos (9).

Algo más comedido se muestra Bunge en su crítica al filósofo austro-británico Karl Raimund Popper (1902-1994), un pensador imprescindible desde que se insertó en la elite filosófica de su tiempo (la Ilamada Escuela de Frankfurt) y más tarde (años 1950 y 1960) en los famosos seminarios en la London School of Economics. La idea central de Popper se sintetiza en que la ciencia progresa a través de refutaciones y no de confirmaciones, y su argumento consiste en que una idea científica jamás podrá ser demostrada como cierta, debido a que por muchas observaciones que parezcan convalidarla, siempre puede ser incorrecta, mientras que un único experimento u observación que la contradiga basta para demostrar su falsedad (10). A eso agregaba que las teorías siempre pueden 
criticarse de forma racional y objetiva, buscando y extirpando sus errores de manera que se allane el camino para distinguir entre teorías mejores y peores. Bunge acude a un extraño símil vinculado con las hortalizas para rebatir estas nociones. Textualmente escribe:

\footnotetext{
Contrariamente a lo que enseñaba Karl Popper, el conocimiento no avanza refutando conjeturas, sino encontrando verdades, o sea, confirmando conjeturas, especialmente hipótesis sobre mecanismos de acción. Análogamente, el cultivo de hortalizas involucra el desmalezamiento, pero lo que comemos son hortalizas, no malezas. (1 p.90)
}

Confieso que no he conseguido entender la analogía (zanálogamente a qué?) y que no hallo ningún indicio en ella para derribar el celebérrimo punto de vista popperiano.

\section{FASCINACIÓN POR LOS MEDICAMENTOS}

En realidad la seducción acrítica por los medicamentos va más allá que esta pincelada sobre los psicofármacos. "Hay dos farmacopeas: la eficaz para ricos, y la ilusoria para pobres" resume Bunge (1 p.124). Es correcta la idea que quiere transmitir el autor, pues apunta a una verdad bien sedimentada: la investigación farmacológica se centra en los fármacos que tienen un mercado poderoso y desdeña las dolencias propias de los países subdesarrollados, cuyos habitantes no pueden pagar fármacos caros. Pero también se alinea con una convicción extendida pero errónea, ya que da por sentado que los fármacos que compran "Ios ricos" son eficaces. La refutación de esta falacia no resulta difícil; basta profundizar un poco en la vasta documentación al respecto.

Durante el seminario internacional realizado con el sugerente título de "El incentivo oculto en la medicina", que se llevó a cabo en Mendoza, Argentina, en 2002, el presidente de la Asociación Médica Federal, Miguel Matta, ironizaba diciendo: "en Argentina, por ejemplo, existen doce mil presentaciones farmacológicas, 12 veces más que en Suiza. Seguramente la población suiza está muy enferma por no disponer de nuestro abanico terapéutico" (11). El total de especialidades y presentaciones farmacológicas registradas en España es incluso un 50\% mayor: ascendía a 18.250 en 2004 (12); estos medicamentos producen anualmente unos 19 millones de efectos adversos y provocan la muerte a 6.500 pacientes $(13,14)$.

Lamentablemente, los médicos toman parte activa en el accionar que desemboca en tan trágica estadística (15); contribuyen también a la "pérdida de muchos billones de dólares para nuestras economías nacionales cada año", como hacía notar Gøtzsche en su artículo de la revista Trials (6).

\section{EL LEGADO DE IVAN ILLICH}

La realidad descrita en la sección precedente es hoy bien conocida. Cuatro décadas atrás solo se atisbaba de manera más bien difusa; hasta que irrumpió el austríaco Ivan Illich. Resulta asombrosa la descalificación a su obra a cargo de Bunge en el libro que nos ocupa: "Sus escritos son malos para la salud individual y para la salud pública" (1 p.167).

Considero que el libro Némesis Médica (16) es un verdadero clásico, que desempeñó un papel esencial en el momento histórico en que vio la luz. Conmovió los cimientos de la hegemonía y la prepotencia (muchas veces irresponsable) de la clase médica, y lo hizo por medio de una brillante exposición de ideas que nadie se había atrevido a desarrollar con tal claridad y con tamaño desenfado. No es admisible que se borre con un par de oraciones a uno de los analistas más originales de los problemas asociados a la práctica médica en la segunda mitad de la última centuria. Según comunica Bunge, este libro ilustra cómo predomina la ideología que genera "desconfianza a la ciencia y en general a la razón" (1 p.195). Sin embargo, tal visión ignora que no era la ciencia, ni mucho menos la razón, las que se ubicaban en el banquillo de los acusados; por el contrario, por conducto de una racionalidad impecable y de un poderoso armamento científico, allí estaban -debido a su irracionalidad y a que daban la espalda a los datos que aportaba la ciencia- las desviaciones que afectaban a la práctica sanitaria de su tiempo.

Bunge reacciona airadamente contra Illich por haber "acusado a la medicina de 'promover enfermedades" (1 p.167). Acusar a la medicina 
como tal, sería una estupidez en la que, desde luego, no incurre Illich. Eso sería como acusar a la sideromecánica de los muertos que ocasionan las armas. Acusó al modo en que fue institucionalizándose la medicina, al establishment médico. Denunciar las aberraciones que aquejan a la medicina no puede confundirse con escribir "contra la medicina", como tendenciosamente dice Bunge (1 p.166), máxime cuando Illich reconoce reiteradamente muchísimas de sus conquistas.

Némesis Médica comenzaba afirmando: “La medicina institucionalizada ha llegado a ser una grave amenaza para la salud" (16 p.9). Illich fue en ese terreno, sobre todo, un visionario. Se adelantó a su tiempo al caracterizar y fundamentar un fenómeno que no ha cesado de crecer alarmantemente: la invención de enfermedades (17) y la medicalización de la sociedad (18). Bunge es insuficiente al definir este último concepto como "la tentativa de hacer pasar los problemas sociales por problemas médicos" (1 p.167). En cierto sentido es así pero, como explica e ilustra brillantemente Teresa Forcades (19), lo que suele pretenderse es más bien que los problemas de origen social se encaren a través de intervenciones médicas sobre el individuo que los padece. Y por otra parte, la medicalización de la sociedad incluye un importante problema que queda fuera de su definición: la conversión de los procesos o rasgos naturales en enfermedades (calvicie, vejez, disfunción eréctil en edades avanzadas, timidez, menopausia, etc.) (20-25) y el manejo de acontecimientos vitales como si fueran dolencias (el nacimiento, la infelicidad o el duelo a raíz de la muerte de seres queridos) $(26,27)$, con la consiguiente intervención -innecesaria y muchas veces iatrogénica- de acciones terapéuticas.

A modo de ejemplo del rigor que destila todo el libro de Illich, me tomaré la libertad de citar uno solo de sus párrafos (las ocho referencias voladas que figuran en este párrafo corresponden a respectivos estudios que Illich cita y comenta a pie de página):

En los Estados Unidos, el volumen del negocio de medicamentos se ha multiplicado por un factor de 100 durante el siglo actual: ${ }^{105}$ 20 mil toneladas de aspirinas se consumen cada año, casi 225 tabletas por persona ${ }^{106}$. En Inglaterra, una de cada diez noches de sueño es inducida por un medicamento hipnótico y el $19 \%$ de las mujeres y el $9 \%$ de los hombres toman por prescripción un tranquilizante durante cualquier año dado. ${ }^{107}$ En los Estados Unidos, los productos que actúan sobre el sistema nervioso central son los que se difunden con mayor rapidez en el mercado farmacéutico, ya representan el $31 \%$ del total de las ventas. ${ }^{108}$ La dependencia respecto de los tranquilizantes recetados ha aumentado un $29 \%$ desde 1962, periodo durante el cual el consumo per cápita de alcohol solo aumentó un $23 \%$ y el consumo calculado de opiáceos ilegales, un $50 \% .{ }^{109}$ En todos los países se obtiene una cantidad significativa de estimulantes y sedantes sin recurrir al médico. ${ }^{110} \mathrm{La}$ toxicomanía medicalizada ${ }^{111}$ ha superado en 1975 a todas las formas escogidas más festivas de crear bienestar. ${ }^{112}$ (16 p.95)

"Lo que era radical en 1974 es, en cierto sentido, hoy una realidad cotidiana", reconocía el afamado editor del British Medical Journal, Richard Smith, en un editorial escrito 30 años después de la publicación de Némesis Médica (28). Smith exalta el poderío del libro como algo "imposible de minimizar" y destaca su notable capacidad de anticipación; concluye recomendando su estudio a todo médico o estudiante de medicina. En efecto, Illich se adelantó a denunciar el Ilamado "disease mongering", según se le conoce actualmente.

Este fenómeno ha sido profusamente tratado en años recientes. Bunge hace una tibia pero correcta alusión a las enfermedades inventadas al referirse a "las deficiencias hormonales y enfermedades mentales fabricadas por ciertos laboratorios farmacéuticos" (1 p.66). También menciona la homosexualidad que, "pese a no ser más que una desviación de la norma estadística, figuró hasta 1974 en la lista de trastornos mentales de la American Psychiatric Association". No creo personalmente que esta inclinación sexual sea ninguna desviación, ni estadística ni de ningún tipo (b). Como no lo es ser superdotado o zurdo o pelirrojo. Ignoro qué es "una norma estadística", pero en castellano una "norma" es una regla que se debe seguir o a la que se deben ajustar las conductas. Si fue considerado un trastorno psiquiátrico, o es aún conceptuado en algunos ámbitos como una desviación, no se debe a rasgo estadístico alguno sino 
a que se incurre en un gran disparate científico o a que prevalece un vituperable prejuicio cultural.

Ahora bien, el problema no se circunscribe a unas pocas enfermedades imaginarias sino que tiene enorme calado; basta acudir a la profusa literatura sobre el tema (29-32) donde se denuncia el monto abrumador de sus manifestaciones. En cualquier caso, esa sólida y consistente disección contemporánea del fenómeno es heredera directa de la obra seminal de Ivan Illich.

Por otra parte, resulta insólito que Bunge afirme que en ella se sostuvo que "la medicina causa más enfermedades de las que cura" y agregue olímpicamente que lo hizo sin el más mínimo dato objetivo de respaldo. No dice que la obra esté deficientemente apoyada en investigaciones, lo cual sería igualmente sorprendente, aunque pudiera admitirse en la medida que la insuficiencia es algo relativo y subjetivo. No, Bunge afirma que no tiene "ni el menor sostén empírico" (1 p.195). Uno se pregunta si Bunge leyó realmente la obra o no la recuerda bien, pues uno de los rasgos que más impresiona es la anonadante cantidad de datos que aporta Ivan Illich para sustentar su discurso (c), tal y como se aprecia en el párrafo reproducido. Tengo la impresión de que, precisamente, debido a que sabía que el libro sería motivo de furiosas reacciones por parte del estamento médico, Illich puso especial celo en refrendar cada aseveración con datos contundentes y precisos.

\section{Visitadores médicos, manipulación y medicocentrismo}

Sorprenden algunos enfoques del libro que solo serían explicables por una notable falta de información. El ejemplo más desconcertante emerge cuando se repara en que Bunge considera que, para estar al corriente de los avances de la medicina, el médico ha de ilustrarse a través de los "visitadores médicos" (1 p.184) por constituir ellos, a su juicio, una de las tres fuentes nutricias para estar al día (las otras son la lectura de literatura y la asistencia a seminarios y congresos). Si bien en el Prefacio sugería que el médico debe estar siempre alerta para filtrar la información que ellos brindan, tal precaución desaparece en el cuerpo del libro. No es para nadie un secreto que la única encomienda asignada a tales trabajadores -asalariados por lo general de las transnacionales del medicamento- es conseguir que se prescriban las mercancías que fabrican sus empleadores, existan o no estudios rigurosos que los pongan en cuestión, con harta frecuencia mediante sobornos directos o encubiertos (33).

Es bastante obvio que no cabe recomendar poner las gallinas al cuidado del zorro, ni siquiera en caso de que estemos alertas sobre su conducta. Personalmente, creo que esa es una pésima vía para estar al tanto de los progresos científicos, la valoración de los cuales exige, ante todo, un clima de transparencia, honradez y debate. Por muchos desatinos en que pueda haber incurrido el trascendente filósofo francés Michel Foucault (d), su reflexión según la cual la verdad termina siendo en el imaginario colectivo aquella que establece el poder, es dramáticamente atinada y actual. El asfixiante dominio del poder mediático y, de hecho, de las empresas a las que este se somete, termina por imponer "la verdad". El personal sanitario -aquel que prescribe en particular, con su enorme potencial de generar ganancias a los laboratorios- está lejos de permanecer inmune a tal manipulación.

Las reflexiones de Bunge están matizadas por un medicocentrismo que atraviesa, directa o implícitamente, todo el material. Se trata de un libro "para médicos", es cierto, tal y como anuncia desde el título de la obra. Sin embargo, para encarar con rigor el examen de las tareas y de los problemas éticos y filosóficos que se vinculan con los médicos, ha de contemplarse al numeroso colectivo de profesionales que interactúan con ellos. Tanto en materia de diagnóstico como de accionar terapéutico, preventivo o rehabilitador, las ciencias médicas actuales demandan del concurso interdisciplinario de muchos otros especialistas: cibernéticos, físicos, bioquímicos, enfermeros, biotecnólogos, odontólogos, estadísticos, fisiatras, psicólogos, farmacéuticos e ingenieros; e incluso de abogados, periodistas, trabajadores sociales, documentalistas y economistas, por solo mencionar algunas disciplinas.

En lugar de encarar la ímproba tarea de señalar aquellos muchos puntos en que las omisiones pudieran ser notables, resulta más expresivo que me detenga en un punto en que ese anacronismo se manifiesta de manera más flagrante: el de los juicios que emite sobre la Enfermería. 
Aparte del lenguaje patriarcal que emplea (siempre se refiere a "médico" y "enfermera"; lo cual es sorprendente ya que, si bien la desinencia masculina es omnicomprensiva, lo cual podría justificar que no emplee jamás el sustantivo "médica", esa condición también la tiene el vocablo "enfermero", término que, sin embargo, tampoco usa nunca), todas sus consideraciones responden al modelo tradicional que considera al enfermero como un mero auxiliar de la consulta médica y reivindica una endogamia elitista para el cuerpo médico. Vale la pena citarlo in extenso:

En años recientes han aparecido facultades de enfermería que ofrecen títulos de postgrado, y algunos de cuyos profesores obtienen subsidios de investigación [...] Sin duda, era hora de que se reconociese la importancia de la enfermería. Pero ¿se beneficia a esta artesanía separándola de la medicina y queriendo hacerla pasar por ciencia? Y ise le hace un favor al paciente si se reemplaza a su médico por una enfermera o si se subvierte una jerarquía asistencial basada en una jerarquía de saberes? En otros campos, los artesanos son reconocidos profesionalmente sin pompa académica. Por ejemplo, todos respetamos a buenos progenitores, constructores, electricistas, técnicos de rayos $X$, pilotos, técnicos de laboratorio y cineastas, pero nadie ha propuesto conferirles títulos académicos avanzados. ¿Magister en paternidad? ¿Doctor Charlie Chaplin? Los artesanos no aprenden principalmente en libros sino en el trabajo, y son útiles cuando trabajan bajo la supervisión de expertos con conocimientos amplios, profundos y al día con la literatura especializada. La tecnología médica propiamente dicha [...] es otra cosa: requiere no solo ingenio y habilidad manual, sino también mucho conocimiento científico. (1 p.189)

En un acto de extemporánea pleitesía a la clase médica, Bunge señala la inconveniencia de violentar la jerarquía asistencial (es decir, que el médico deje de ser conceptuado como superior al enfermero), ya que esta se fundamenta en la jerarquía de conocimientos o saberes (o sea, en que los conocimientos de los médicos son superiores a los de los enfermeros). No es difícil comprender que no se trata de quién sabe más cuando la sabiduría concierne a zonas diferentes, aunque complementarias (34). Por otra parte, creo que nadie, al dar estatuto académico a la Enfermería, propone que los médicos desaparezcan y solo queden a cargo de la asistencia los especialistas de aquella disciplina. Sin embargo, Bunge parece dar por sentado que ello pudiera ocurrir, de modo que considera necesario Ilamar la atención sobre tan peligrosa deriva para los pacientes.

Su discurso puede sintetizarse así: respetemos a los enfermeros, pero poniéndolos en su lugar; no debemos olvidar que son simples artesanos (e) que no tienen nada que investigar y que han de trabajar bajo la supervisión de quienes realmente tienen conocimientos amplios y profundos. Les corresponde ser obedientes y dedicados a su encomienda subalterna.

Si fuera por él, deberían quizá eliminarse las centenas de revistas científicas de enfermería registradas en Scopus y Medline, así como los miles de libros sobre esta disciplina que actualmente se producen. La literatura científica sería innecesaria para aquellos a los que solo toca ser habilidosos y a lo sumo ingeniosos, tal y como le ocurre a los albañiles.

Ignora que desde hace muchos años los cuidados de enfermería tienen sus propias funciones, que abarcan a la promoción, el mantenimiento y la recuperación de la salud, y son cruciales no solo para el manejo de dolencias y discapacidades sino también para su prevención (35). Anclado en una concepción hace ya mucho superada, Bunge nos aclara que ellos no tienen sus propios sistemas de saberes y defiende sobre esas bases una subordinación operativa e intelectual que retrotraería a la atención sanitaria a los modelos teórica y prácticamente dominantes a comienzos del siglo XX. En efecto, en 1902 el Doctor por la University of Glasgow, Joseph McGregor Robertson afirmaba: "Una enfermera debe empezar su trabajo con la idea, firmemente implantada en su mente, de que ella no es más que un instrumento que recibe instrucciones del médico; ella no ocupa una posición independiente en el tratamiento de una persona enferma" (36). Y la enfermera diplomada (Registered Nurse) de Kentucky, Sarah Dock, escribía en 1917:

Al margen de cuán competente pueda ser, ella jamás será una enfermera confiable hasta 
que no obedezca sin cuestionarse nada. La primera y más útil crítica que jamás recibí de un médico fue cuando él me dijo que de mí se esperaba que fuera una simple máquina inteligente para cumplir sus órdenes. (37) [traducción nuestra]

Pero algunas de las ideas de Bunge nos llevan, en rigor, a un siglo y medio atrás; basta reparar en la forma en que Florence Nightingale ironizaba al respecto en 1860 :

Ningún hombre, ni siquiera un médico, ha dado una definición diferente de lo que una enfermera debe ser fuera de que ha de conducirse de manera "dedicada y obediente". Esta definición sería igualmente válida para un cochero. También pudiera serlo para un caballo.

(38) [traducción nuestra]

Las habilidades y el ingenio son desde luego necesarios, como en cualquier otra profesión, la médica incluida; pero sería interminable conformar la lista de tareas de enfermería que exigen de decisiones independientes a la hora de accionar sobre un paciente, así como de conocimientos altamente especializados y subespecializados y, por tanto, no susceptibles de ser realizados eficientemente por nadie que no esté versado y científicamente actualizado en esos dominios.

\section{TEORÍA DE PROBABILIDADES Y SUBJETIVIDAD}

Aparte de algunos errores puntuales y de opiniones que, a mi juicio, dimanan de un pensamiento rígido o atascado en visiones conservadoras, la mayoría de las observaciones críticas hasta aquí realizadas apuntan a exageraciones, simplificaciones o banalizaciones de Bunge. Pero no es ese el caso cuando el autor se interna en el mundo de las probabilidades y el pensamiento bayesiano: en ese marco se muestra especialmente errático. El examen detenido de los desatinos de Bunge en esta materia exige consideraciones técnicas que desbordan la presente reflexión y puede ser consultado en otro sitio (39), pero me permitiré bosquejar la esencia de tales errores.
Sus yerros fundamentales -sustento de toda su incursión en el tema- residen, por una parte, en no comprender que arbitrariedad y subjetividad son dos conceptos totalmente diferentes y, por otra, en considerar que la probabilidad es una noción exclusivamente aplicable en procesos intrínsecamente aleatorios (entendidos en el sentido de que no se rigen por un mecanismo causal). Repasemos brevemente ambos asuntos.

"Por ser subjetivas, las probabilidades bayesianas son arbitrarias" afirma textualmente (1 p.99). La arbitrariedad y el capricho comprometen cualquier discurso científico; pero es bien sabido que la subjetividad es inevitable en la ciencia, como ha sido casi universalmente admitido $(40,41)$. Tal realidad abarca al ámbito de las probabilidades. A partir de ese equívoco, Bunge atribuye a los estadísticos bayesianos una conducta antojadiza. "El bayesiano asigna las probabilidades que se le antojen" (1 p.99), afirma, y un par de páginas más adelante va más lejos y asevera tajantemente que "no cabe hablar de probabilidades en medicina" (1 p.101).

La idea presente en la interpretación subjetiva de la probabilidad es que, ante un fenómeno aleatorio o concebido como tal para resolver determinado problema (en el sentido de que puede verificarse o no y que resulta imposible conocer de antemano cuál de esos desenlaces se producirá), se asigna, de forma implícita o explícita, una probabilidad que representa el grado de confianza o creencia que se tiene en la ocurrencia de ese hecho. Bajo este enfoque, la probabilidad no está determinada de antemano, sino que se establece en función de la información -cuantitativa o no- con que se cuente acerca de la situación, así como de la forma en que se valore dicha información.

La diferencia esencial con la interpretación frecuentista radica en que la asignación de valores, aunque condicionada por la información de que se disponga, es propia de cada observador particular, sin que las opiniones de varios analistas tengan que coincidir exactamente. Bajo el enfoque frecuentista, la probabilidad de cierto suceso es un número único e ideal (concretamente, el límite de la razón entre el número de veces que dicho suceso ocurre y el de aquellas en que se lleva adelante el proceso que pudiera producirlo, cuando este último número tiende a infinito), y lo que puede variar son las estimaciones que hacemos de ella. 
Cuando se dice que la probabilidad de que Brasil gane a Corea del Norte, en un partido que disputarán en el Campeonato Mundial, es igual a 0,95 , la de que empate a 0,04 y la de que pierda a 0,01 , no se han elegido esos números de una tabla de números aleatorios ni en función de preferencias estéticas. Se han fijado sobre una base subjetiva -el desempeño de sus delanteros en partidos recientes, el número de jugadores que ya tienen una tarjeta amarilla, el valor de los porteros en el mercado, los resultados obtenidos en partidos recientes y la calidad de los contrincantes en dichos partidos, etc.-, pero no arbitraria (f). Si las casas de apuestas fijaran las probabilidades en que se basan estos premios a su antojo o guiadas por preferencias estéticas, en lugar de obtener cuantiosas ganancias, simplemente quebrarían.

Obviamente, para que esta interpretación pueda ser aplicada exitosamente en un marco operativo, es menester que quienes se acogen a ella mantengan un alto grado de racionalidad en la asignación de probabilidades. Si se quiere hacer inferencias válidas, los valores que se determinen no pueden ser fruto del capricho o del "antojo" de quien los fija. Una vez asignados por esa vía los grados de confianza que se tengan en la ocurrencia de los sucesos, si los valores correspondientes satisfacen los axiomas de Kolmogorov (42), ya se opera con esas probabilidades como con cualquier otra manera de definirlas que también cumpla aquellos axiomas.

"Solo los hechos al azar y los escogidos al azar tienen probabilidades" (1 p.100), sostiene Bunge. Es ciertamente difícil o imposible de interpretar el concepto de que un hecho tenga probabilidad. Los eventos no tienen probabilidades; se les atribuyen probabilidades de ocurrencia por una u otra vía si se considera que ello puede ser fructuoso. No es un detalle baladí sino medular: mientras lo primero apunta a un rasgo que sería presuntamente intrínseco a determinados "hechos", lo segundo es una convención que por lo general se adopta con fines operativos.

Bunge comunica que la interpretación bayesiana no se adecua a las ciencias de la salud. Por sorprendente que resulte, tal convicción se basa en que en estas ciencias no prima la diversidad de opiniones. Los pacientes y los médicos, según él, afortunadamente saben que "si hay diferencias de opinión acerca de un tratamiento o una diagnosis, suele recabarse la opinión de un tercero o de un panel de expertos, de quienes se espera no solo opinión sino también argumentos fundados en las ciencias biomédicas" (1 p.101). Lo que no capta Bunge es que, en cualquier caso, hablamos de una opinión que, como tal, habrá de involucrar inexorablemente cierto grado de subjetividad para ser conformada, a veces muy grande, a veces menor. No puede ser de otro modo: las ciencias biomédicas están plagadas no solo de incertidumbres, verdades provisionales, controversias y dudas, sino que con mucha frecuencia dan por cierto aquello que no lo es (43). Y finalmente, los "argumentos fundados" no son privativos de las ciencias biomédicas; también están presentes en la asignación de probabilidades, y tal asignación puede adoptarse igualmente tras la consulta con otro especialista o apelando a un panel de expertos.

Ahora bien, el meollo de este debate reside en lo siguiente: ¿sobre qué bases se puede aceptar o no un enfoque metodológico dado en el contexto de la solución de un problema? En el marco que nos ocupa, esto se traduce en la pregunta: ¿cuál pudiera ser el árbitro que concede o no validez a la asignación de probabilidades a los eventos no intrínsecamente aleatorios?

\section{LA LEGITIMIDAD DE LOS MODELOS}

Examinemos más detenidamente la afirmación según la cual "no es legítimo asignar una probabilidad a todo hecho. Solo los hechos al azar y los escogidos al azar tienen probabilidades" (1 p.100).

Un modelo, entendido como una idealización o representación de la realidad, que procura simplificarla para poderla examinar mejor y luego aplicar sus derivaciones en la solución de un problema en el contexto de complejidad que la inspiró, solo admite una valoración pragmática. Dicho de otro modo: dado que su construcción responde a que se procura resolver un problema por su conducto, el modelo solo puede deslegitimarse en caso de que no contribuya a resolver el problema que ha llevado a concebirlo.

Es la misma lógica que puede aplicarse a la hora de valorar la legitimidad de un modo de conducirse con vistas a resolver un problema práctico en general. Dejando a un lado la dimensión ética 
en su valoración, que es otro tema, la legitimidad de esa conducta se supedita siempre al grado en que permita resolver el problema que ha llevado a desplegarla. No puede ser "ilegítima" si es útil, ni "legítima" si es estéril.

Obviamente, si Brasil vence es por una causa (porque el desarrollo del partido produce que convierta más goles que Corea del Norte); y si Obama gana las elecciones será por determinadas causas que conducen a que obtenga más votos que sus contrincantes. El desenlace es debido a tales causas, sean las que sean; pero el proceso que lo determina permite manejar dicho desenlace como un suceso aleatorio debido a que no existe manera alguna de identificarlo con certeza de antemano, aunque conozcamos el valor de algunas de las variables que pudieran influir en él.

Si las casas de apuestas se sometieran al dogma bungeano, no tendrían las ganancias millonarias que consiguen (44); y si Nate Silver hubiera obedecido ese ucase metodológico, no hubiera tenido el sensacional éxito en su vaticinios electorales en EE.UU. (45).

Bunge reniega del empleo de la teoría de probabilidades incluso en situaciones donde ni siquiera interviene la subjetividad para su definición. Llega a decir que Ilamar "probabilidades" a las frecuencias relativas que manejan los epidemiólogos "es doblemente errado: porque las frecuencias son propiedades colectivas y porque el uso de probabilidades solo se justifica con referencia a procesos aleatorios, dado que los procesos subyacentes tienen raíces causales" (1 p.102) . Y luego vuelve sobre el tema al considerar que:

Es verdad que se habla a menudo de la "probabilidad" de que tal tratamiento cure tal mal, pero este uso del concepto de probabilidad es incorrecto, porque el concepto en cuestión es teórico, no empírico. Las "probabilidades" de que se habla en medicina y en epidemiología son en realidad frecuencias relativas, y éstas no están necesariamente (lógicamente) relacionadas con el azar. (1 p.142)

De aquí concluye que "Ios médicos [...] harán bien en limitarse a manejar frecuencias estadísticas" (1 p.142), sin considerarlas probabilidades. $\mathrm{Si}$ las frecuencias relativas no pudieran considerarse probabilidades -un dictum inventado por Bunge (g) - no se podría, por poner un solo ejemplo, calcular la esperanza de vida, un parámetro capital de la salud pública contemporánea, basado en la teoría de probabilidades.

Para concluir, señalo que la rotunda negación de Bunge al empleo de la teoría de probabilidades en el ámbito de la salud nos remite a la polémica que se produjo a mediados del siglo XIX, cuando el cirujano-urólogo Jean Civiale (1792-1867) intentó, mediante la utilización de la estadística, tomar partido entre dos tratamientos para operar los cálculos de vejiga. El debate, con participación de prestigiosas figuras, como Siméon Poisson, se verificó en el marco de la Academia de Ciencias de París. Uno de los comisionados, el español Risueño de Amador (1802-1849), sentenció algo que parece salido de la pluma de Bunge: "El cálculo de probabilidades nos parece demasiado imperfecto, incluso matemáticamente hablando, porque los matemáticos no se ponen de acuerdo en algunos aspectos importantes de la teoría. Su aplicación en la medicina, sobre todo en la terapéutica, es anticientífica" (46). Finalmente, sin embargo, la Academia se decanta a favor de la metodología de análisis de Civiale expresando: "La medicina es una ciencia de observación exactamente como las demás, las estadísticas y el cálculo de probabilidades tiene algo que decirnos en cuanto a las conclusiones que debemos sacar y con qué grado de confianza debemos hacerlo" (47). 


\section{NOTAS FINALES}

a. Ha de señalarse, sin embargo, que uno se ve tentado a hacerlo. Por ejemplo, desconcierta que en el libro no se haga la menor mención a Internet ni a las nuevas tecnologías de la información y la comunicación, como si ellas no tuvieran una enorme y creciente gravitación en los planos práctico, conceptual, axiológico, tecnológico y, consecuentemente, filosófico, dentro de la praxis sanitaria.

b. Anteriormente ya se había insistido en esta idea. Allí Bunge no alude a la homosexualidad como rasgo sino a los homosexuales propiamente dichos y explica que, mientras antaño los homosexuales eran vituperados, "hoy son considerados meras desviaciones estadísticas" (1p.50).

c. De hecho, las notas que los contienen abarcan aproximadamente la mitad de todo el volumen. d. La trascendencia del autor de Las palabras y las cosas está más allá de toda duda razonable, aunque también su obra resulta en buena medida banalizada por Bunge quien, de paso, desliza no sin cierta maledicencia, que nada agrega, que si Foucault "hubiera comprendido que 'la promiscuidad es mala para la salud', no hubiera muerto de SIDA" (1 p.65).

e. Es decir, una "persona que ejercita un arte u oficio meramente mecánico", según el significado que le da nuestra Academia de la Lengua al término "artesano".

f. Dicho sea de paso, esas dos selecciones no se habían enfrentado jamás antes, de modo que sería imposible realizar una estimación frecuentista.

g. Ignoro si algún filósofo, epistemólogo o matemático lo comparte, pero no conozco a ningún otro.

\section{REFERENCIAS BIBLIOGRÁFICAS}

1. Bunge M. Filosofía para médicos. Barcelona: Gedisa; 2012.

2. Vargas M. La civilización del espectáculo. Lima: Alfaguara; 2012.

3. Onfray M. Freud: El crepúsculo de un ídolo. México DF: Taurus; 2011.

4. Gøtzsche PC. Big pharma often commits corporate crime, and this must be stopped. British Medical Journal. 2012;345:e8462. DOI:10.1136/ bmj.e8462.

5. Breggin P, Breggin GR. Talking back to prozac: what doctors won't tell you about today's most controversial drug. New York: St. Martin's Paperbacks; 1994.

6. Gøtzsche PC. Why we need easy access to all data from all clinical trials and how to accomplish it. Trials. 2011;12:249. DOI:10.1186/1745-6215-12-249.

7. Laurance J. Antidepressant drugs don't work: official study. The Independent [Internet]. 6 feb 2008 [citado 10 feb 2013]. Disponible en: http:// www.independent.co.uk/life-style/health-and-fa- milies/health-news/antidepressant-drugs-udontuwork-ndash-official-study-787264.html.

8. Lacasse JR, Leo J. Serotonin and depression: a disconnect between the advertisements and the scientific literature. PLoS Medicine [Internet]. 2005 [citado 5 feb 2013];2(12):e392. Disponible en: http://www.plosmedicine.org/article/ info:doi/10.1371/journal.pmed.0020392.

9. Carey B, Harris G. Antidepressant may raise suicide risk. The New York Times [Internet]12 may 2006 [citado 5 feb 2013]. Disponible en: http:// www.nytimes.com/2006/05/12/health/12depress. html? $r=0$.

10. Banegas JR, Rodríguez-Artalejo F, Rey-Calero J. Popper y el problema de la inducción en epidemiología. Revista Española de Salud Pública. 2000;74(4):327-339.

11. Se realizó en Mendoza el V Congreso Argentino de Salud. Revista Médicos [Internet]. 2002 [citado 5 feb 2013];(22):28-30. Disponible en: http://www.revistamedicos.com.ar/numero22/pagina28.htm.

12. Perancho I. El bolsillo de los españoles notará poco la rebaja del precio de los medicamentos. 
El Mundo [Internet] 27 nov 2004 [citado 5 feb 2013]. Disponible en: http://www.elmundo.es/ salud/2004/596/1101510010.html.

13. Sanchís I. Los ricos mueren por exceso de atención médica. La Vanguardia [Internet] 7 feb 2013 [citado 10 feb 2013]. Disponible en: http://www.lavanguardia.com/ lacontra/20130207/54365145790/la-contra-juangervas.html.

14. Gérvas J, Pérez M. Sano y salvo (y libre de intervenciones médicas innecesarias). Barcelona: Los Libros del Lince; 2013.

15. Kassirer JP. On the take: how medicine's complicity with big business can endanger your health. New York: Oxford University Press; 2005.

16. Illich I. Némesis médica: la expropiación de la salud. México: Joaquín Mortiz; 1978.

17. Blech J. Los inventores de enfermedades. Cómo nos convierten en pacientes. Barcelona: Destino; 2005.

18. Conrad P. The medicalization of society: on the transformation of human conditions into treatable disorders. Baltimore: The Johns Hopkins University Press; 2007.

19. Forcades T. La medicalización de los problemas sociales. Revista Cubana de Salud Pública. 2012;38(Supl):75-78.

20. Szymczak JE, Conrad P. Medicalizing the aging male body: andropause and baldness. En: Rosenfeld D, Faircloth C, editors. Medicalized Masculinities. Philadelphia: Temple University Press; 2006.

21. Carpiano RM. Passive medicalization: The case of viagra and erectile dysfunction. Sociological Spectrum. 2001;21(3):441-450.

22. Ebrahim S. The medicalisation of old age. British Medical Journal. 2002;324:861.

23. Moynihan R. The making of a disease: female sexual dysfunction. British Medical Journal. 2003;326:45.

24. Scott S. The medicalisation of shyness: From social misfits to social fitness. Sociology of Health and IIIness. 2006;28(2):133-153.

25. Bell SE. Changing ideas: The medicalization of menopause. Social Science and Medicine. 1987;24(6):535-552.
26. Shaw I, Woodward L. The medicalization of unhappiness? The management of mental distress in primary care. En: Shaw I, Kauppinen K, editors. Constructions of Health and Illness: European Perspectives. Aldershot: Ashgate; 2004.

27. Márquez S, Meneu R. La medicalización de la vida y sus protagonistas. Gestión Clínica y Sanitaria. 2003;5:47-53.

28. Smith R. Limits to Medicine: Medical Nemesis: The expropriation of Health (book review). British Medical Journal. 2002;324:923.

29. MacTaggart L. Lo que los médicos no nos dicen: los riesgos de la medicina moderna. Barcelona: Terapias Verdes; 2005.

30. Payer L. Disease-mongers: How doctors, drug companies, and insurers are making you feel sick. New York: Wiley and Sons; 1992.

31. Moynihan R, Heath I, Henry D. Selling sickness: The pharmaceutical industry and disease-mongering. British Medical Journal. 2002;324(7342):886891.

32. Moynihan R, Henry D. The fight against disease mongering: Generating knowledge for action. PLoS Medicine. 2006;3(4):e191. DOI:10.1371/ journal.pmed.0030191.

33. Angell M. The truth about the drug companies: how they deceive us and what to do about it. New York: Random House; 2005.

34. Fagin L, Garelick A. The doctor-nurse relationship. Advances in Psychiatric Treatment. 2004;10: 277-286. DOI:10.1192/apt.10.4.277.

35. Escuela Universitaria de Enfermería Santa Madrona. La formación enfermera: estado de la cuestión y perspectiva internacional. Barcelona: Fundació Caixa de Pensions; 2004.

36. McGregor J. The Household Physician. London: Gresham Publishing; 1902.

37. Dock S. The relation of the nurse to the doctor and the doctor to the nurse. American Journal of Nursing. 1917;17(5):394-396.

38. Nightingale F, Skretkowicz V. Florence nightingale's notes on nursing and notes on nursing for the labouring classes: Commemorative edition with historical commentary. New York: Springer Publishing Company; 2010.

39. Silva LC. En defensa de la racionalidad bayesiana: a propósito de Mario Bunge y su "Filosofía 
para médicos" [Internet]. 2013 [citado 2 feb 2013]. Disponible en: http://lcsilva.sbhac.net/Articulos/articulos.htm.

40. Press SJ, Tanur JM. The subjectivity of scientists and the Bayesian approach. New York: Wiley and Sons; 2001.

41. Silva LC, Benavídes A. Apuntes sobre subjetividad y estadística en la investigación. Revista Cubana de Salud Pública. 2003;29(2):170-173.

42. Kolmogorov AN. Foundations of the Theory of Probability. New York: Chelsea Publishing Company; 1956.

43. Ioannidis JPA. Why most published research findings are false? PLoS Medicine. 2005;2(8):e124. DOI:10.1371/journal.pmed.0020124.

44. Morales P. ¿Cuánto ganan las casas de apuestas deportivas? ABC [Internet] 26 may 2012 [citado 5 mar 2013]. Disponible en: http://www. abc.es/20120525/deportes/abci-beneficios-casasapuestas-deportivas-201205220210.html.

45. Garicano L. Son las Matemáticas, estúpido. El País [Internet]. 13 nov 2012 [citado 5 mar 2013]. Disponible en: http://elpais.com/elpais/2012/11/12/ opinion/1352747017_116039.html.

46. Rodríguez-Lázar AF. El cálculo de probabilidades en la polémica médica del siglo XIX: aportaciones españolas. En: Santos del Cerro J, García Secades M, coordinadores. Historia de la Probabilidad y de la Estadística. Madrid: AHEPE; 2002. p.133-151.

47. Almenara J, Silva LC, Benavides A, García C, González JL. Historia de la Bioestadística: La génesis, la normalidad y la crisis. Cádiz: Quorum editores; 2003.

\section{FORMA DE CITAR}

Silva Ayçaguer LC. Reflexiones a raíz de Filosofía para médicos, un texto de Mario Bunge. Salud Colectiva. 2013;9(1):103-116. 